\title{
Engineering Yarrowia lipolytica to enhance lipid production from lignocellulosic materials
}

\author{
Xochitl Niehus ${ }^{2}$, Anne-Marie Crutz-Le Coq ${ }^{1}$, Georgina Sandoval ${ }^{2}$, Jean-Marc Nicaud ${ }^{1}$ \\ and Rodrigo Ledesma-Amaro ${ }^{1,3^{*}}$ (1)
}

\begin{abstract}
Background: Yarrowia lipolytica is a common biotechnological chassis for the production of lipids, which are the preferred feedstock for the production of fuels and chemicals. To reduce the cost of microbial lipid production, inexpensive carbon sources must be used, such as lignocellulosic hydrolysates. Unfortunately, lignocellulosic materials often contain toxic compounds and a large amount of xylose, which cannot be used by Y. lipolytica.

Results: In this work, we engineered this yeast to efficiently use xylose as a carbon source for the production of lipids by overexpressing native genes. We further increased the lipid content by overexpressing heterologous genes to facilitate the conversion of xylose-derived metabolites into lipid precursors. Finally, we showed that these engineered strains were able to grow and produce lipids in a very high yield (lipid content $=67 \%$, titer $=16.5 \mathrm{~g} / \mathrm{L}$, yield $=3.44 \mathrm{~g} / \mathrm{g}$ sugars, productivity $1.85 \mathrm{~g} / \mathrm{L} / \mathrm{h}$ ) on a xylose-rich agave bagasse hydrolysate in spite of toxic compounds.
\end{abstract}

Conclusions: This work demonstrates the potential of metabolic engineering to reduce the costs of lipid production from inexpensive substrates as source of fuels and chemicals.

Keywords: Yarrowia lipolytica, Xylose utilization, Acetyl-CoA, Microbial lipids, Metabolic engineering, Synthetic biology

\section{Background}

Microbial lipids have gained much attention during the last few years due to their potential to replace petroleum as a main source of fuels and chemicals. Lipids and lipidderived molecules can be used in industry to produce biodiesel, biokerosene, pharmaceuticals, nutraceuticals, cosmetics, lubricants, or plasticizers.

Among the most studied microorganisms that are able to produce large amounts of lipids, we find Yarrowia lipolytica, a dimorphic yeast with a known genome and convenient molecular tools for its manipulation [1]. In recent years, the lipid metabolism of this yeast has been

\footnotetext{
*Correspondence: rodrigoledesma@usal.es;

r.ledesma-amaro@imperial.ac.uk

${ }^{3}$ Department of Bioengineering, Imperial College London, London SW7

2AZ, UK

Full list of author information is available at the end of the article
}

rationally modified by metabolic engineering in order to maximize lipid production, mainly using glucose as carbon source. Overexpression of the genes directly involved in fatty acid or TAG synthesis [2,3] and in the supply of NADPH (required cofactor for fatty acid biosynthesis) [4] or deletion of the genes involved in competing pathways, such as beta-oxidation or TAG remobilization [5], has generated strains that can accumulate up to $90 \%$ of their DCW as fatty acids [6] or that can reach a yield of $84.7 \%$ of the theoretical maximal yield [7]. However, the bioconversion of glucose into lipids by $Y$. lipolytica is still a rather expensive process, which limits its industrial viability. Therefore, novel synthetic biology approaches have been recently developed in order to achieve profitable production of biolipids. Such approaches include the following: (1) the engineering of this yeast to enable fatty acid secretion into the culture media, which facilitates 
downstream processes (extraction and separation of the lipids); these downstream processes can be up to $60 \%$ of the total cost of the process [8]; (2) the modification of this yeast in order to use unusual nitrogen or phosphorus sources, which makes it competitively grow in nonsterilized media, limiting the risk of contamination and therefore reducing the cost associated with sterilization [9]; (3) the heterologous expression of enzymes that enable $Y$. lipolytica to produce specific, more expensive final products (either high-value fatty acids or fatty acidderived compounds), such as hydroxylated fatty acids [10] or fatty alcohols [11]; and, finally, (4) the expansion of the range of substrates that this yeast can use as carbon source, allowing it to use waste products or low-cost substrates, such as raw starch [12], xylose [13], fructose [14], galactose [15], or other convenient substrates [16].

In this regard, one of the most preferred substrates for biotechnology is lignocellulosic hydrolysate, which is a raw mixture of nutrients that is normally enriched in C6 and C5 sugars, mainly glucose and xylose, which originate from the degradation of cellulose and hemicellulose from plant biomass. The hydrolysates often have a significant amount of potentially toxic compounds, such as organic acids and furfurals [17], which are usually detrimental to most organisms. Y. lipolytica, often found in contaminated soils, has the potential to resist such harsh conditions. Moreover, Y. lipolytica is able to use glucose very efficiently for the production of lipids. However, the wild-type strains of $Y$. lipolytica cannot use xylose, which can be up to $50 \%$ of the components in lignocellulosic material [18].

Therefore, in the last year, several metabolic engineering approaches have been carried out in order to allow $Y$. lipolytica to use xylose. Interestingly, it seems that different genetic backgrounds lead to variability in the capacity of the strains to use this sugar after engineering. Pold was able to use xylose efficiently after the expression of the heterologous $X D H$ (xylitol dehydrogenase) and $X R$ (xylose reductase) from $S$. stipitis and the overexpression of the native $X K$ (xylulokinase) [13]. The modified strains were optimized to maximize two biotechnological products, lipids and citric acid. However, Polg expressing the three heterologous genes $(X R, X D H$, and $X K)$ was unable to grow on xylose until a starvation adaptation was performed [19]. In the case of Polf background, the overexpression of native genes $\mathrm{XK}$ and $\mathrm{XDH}$ was enough to permit growth in xylose, although its growth was reduced compared to growth on glucose and especially under nitrogen limitation, which is a required condition to overproduce fatty acids [20]. In previous studies, unmodified Po1g, which is unable to use xylose alone, was shown to produce lipids in detoxified hydrolysates supplemented with peptone (rice bran [21] and sugarcane bagasse [22] hydrolysates). However, no strain modified to fully assimilate xylose has yet been tested for lipid production in complex, non-detoxified, and inexpensive lignocellulosic hydrolysates.

In this work, we re-engineer Pold and a lipid overproducer derivative strain to efficiently use xylose, even under nitrogen limitation conditions, using native genes. The modified strains were able to produce high amount of lipids from C5. These strains were further engineered in order to convert xylose into acetyl-CoA using alternative pathways by expressing heterologous genes. These modifications increased lipid production. Finally, the engineered $Y$. lipolytica strains were used to produce lipids from a low-cost lignocellulosic hydrolysate in a fedbatch fermentation, which reached to the highest lipids yields described so far.

\section{Results and discussion}

The overexpression of native genes yIXDH, yIXR, and yIXK is sufficient for efficient growth in xylose

Recently, we found that Y. lipolytica Pold was able to growth in xylose due to the overexpression of $X D H$ and $X R$ from $S$. stipitis and native $X K$. Here, we investigate the ability of native $X D H$ and $X R$ to allow growth in xylose. With this aim, we amplified these two genes and placed them under the control of the strong, constitutive TEF promoter. The modified strain, which contains the overexpression cassettes of $\mathrm{yl} X D H, \mathrm{yl} X R$, and $\mathrm{yl} X K$, was able to grow in xylose as well as the wild type grows in glucose (similar growth rate), and it grew even slightly better than the strain overexpressing the heterologous genes (shorter lag phase) (Fig. 1a, b; Additional file 1: Figure S1). This strain will be referred to in the paper as ylXYL+.

In parallel, we overexpressed the three native genes in a Po1d-derived background that was previously modified to maximize lipid production through overexpression of genes DGA2 and GPD1 from the lipid biosynthetic pathway and deletion of the POX1-6 genes and the TGL4 gene, which are involved in the degradation and remobilization of lipids in Y. lipolytica [14]. The modified strain, named ylXYL+Obese, was able to grow in xylose as a sole carbon source in a similar manner as ylXYL+ (similar growth rate and lag phase) (Fig. 1a, b).

Both ylXYL+ and ylXYL+Obese were able to consume glucose and xylose when a mix of the two sugars was used as a carbon source. However, as previously shown with the heterologous genes [13], both strains present a partially sequential use of the two substrates. Moreover, the obese phenotype shows a higher biomass and a lower xylitol production than ylXYL+ (Fig. 1c for ylXYL+ and Fig. 1d for ylXYL+Obese). 
a

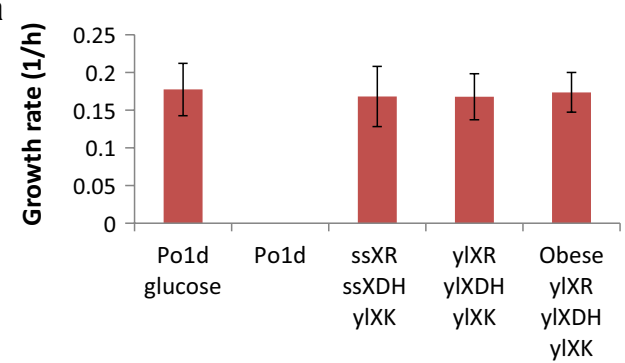

C

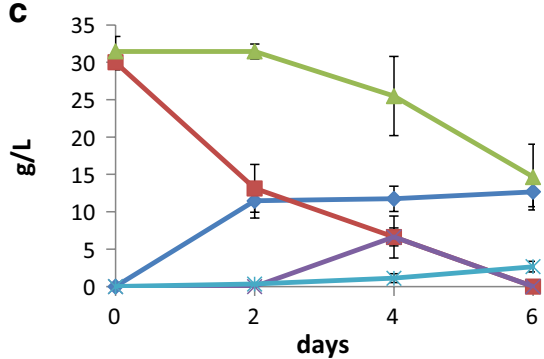

b

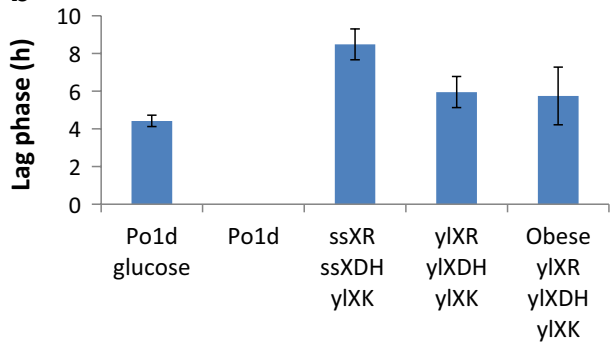

d

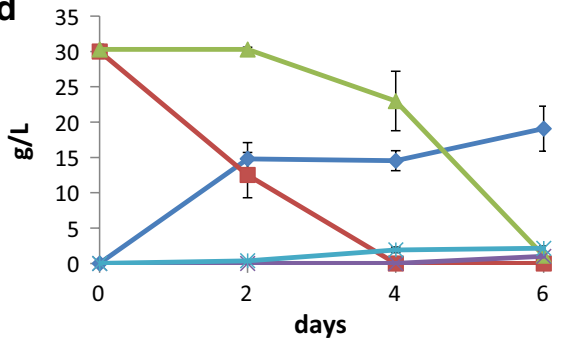

Fig. 1 Growth of different strains in xylose or a mixture of glucose and xylose. Bar plot representation of the growth rate (a) and the lag phase (b) of different $Y$. lipolytica strains. The absence of a bar means that no growth was detected. The values represent the average growth rate (1/h) or lag phase (h) and the standard deviation of at least two replicates growing in microplates. The strain named Po1d is a prototroph-derived strain. Minimal media was used with xylose as a carbon source, unless the carbon source is specified in the figure as glucose. Behavior of the strains yIXYL+ (c) and yIXYL+Obese (d) growing in a mixture of glucose and xylose. The graphics show the consumption of substrates (xylose and glucose, $\mathrm{g} / \mathrm{L}$ ), DCW $(\mathrm{g} / \mathrm{L})$, and production of metabolites (xylitol- $\mathrm{XOH}$ and citric acid-CA, $\mathrm{g} / \mathrm{L}$ )

Yarrowia lipolytica yIXYL+ and yIXYL+Obese can produce lipids from xylose as a sole carbon source

Nitrogen limitation is often required to boost lipid production in $Y$. lipolytica. Therefore, we tested the impact of different carbon/nitrogen ratios in the culture media (YNB20, YNB30, YNB60, and YNB90) on the growth and lipid production capacity of the modified $Y$. lipolytica strains using xylose as a sole carbon source (Fig. 2 and Table 1). After growing both strains in flasks, we found that YNB60 is the most convenient medium for lipid accumulation because a higher or lower ratio decreased lipid accumulation. On the one hand, ylXYL+Obese produces 2 times more lipids than ylXYL+. On the other hand, ylXYL+ produces more xylitol (75-100\% more in YNB60 and YNB90) and citric acid (33-67\% more in YNB60 and YNB90) than ylXYL+Obese (Additional file 1: Figure S2, Table S1). We can here conclude that $y \mathrm{lXYL}+$ Obese is the most convenient strain for the
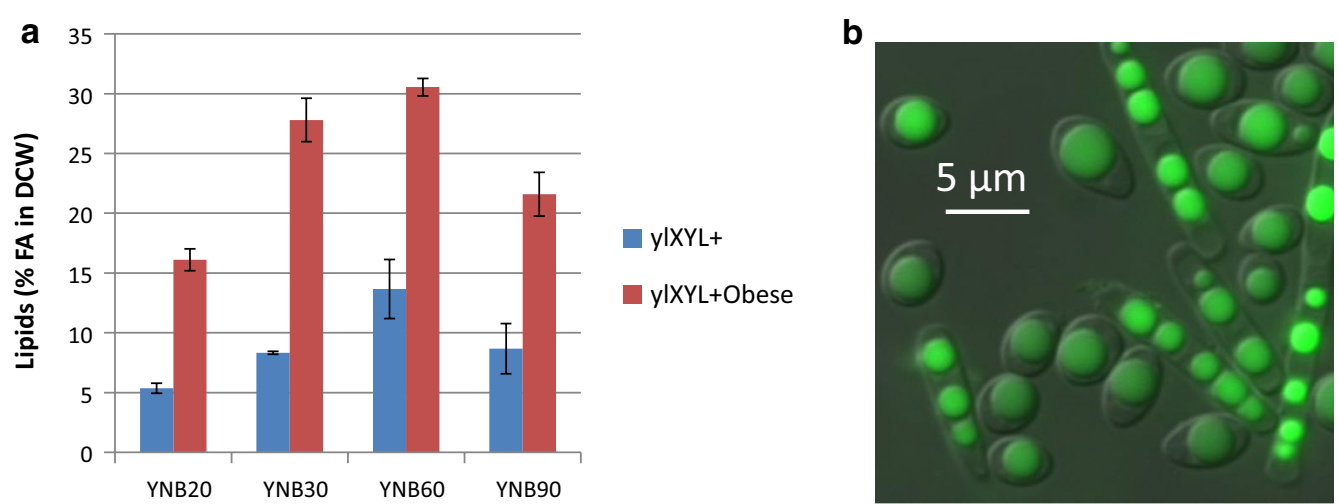

Fig. 2 Lipid production by $y \mid X Y L+$ and yIXYL+Obese strains in xylose. a Amount of lipids (\% of fatty acids in the dry cell weight) produced in the yIXYL+ (blue) or yIXYL+Obese (red) strains grown in varying concentrations of xylose (YNB20, YNB30, YNB60, and YNB90; see "Methods"). b Fluorescence microscopy image showing the cells and their lipids stained with Bodipy in the YNB60 medium with the strain yIXYL+Obese 


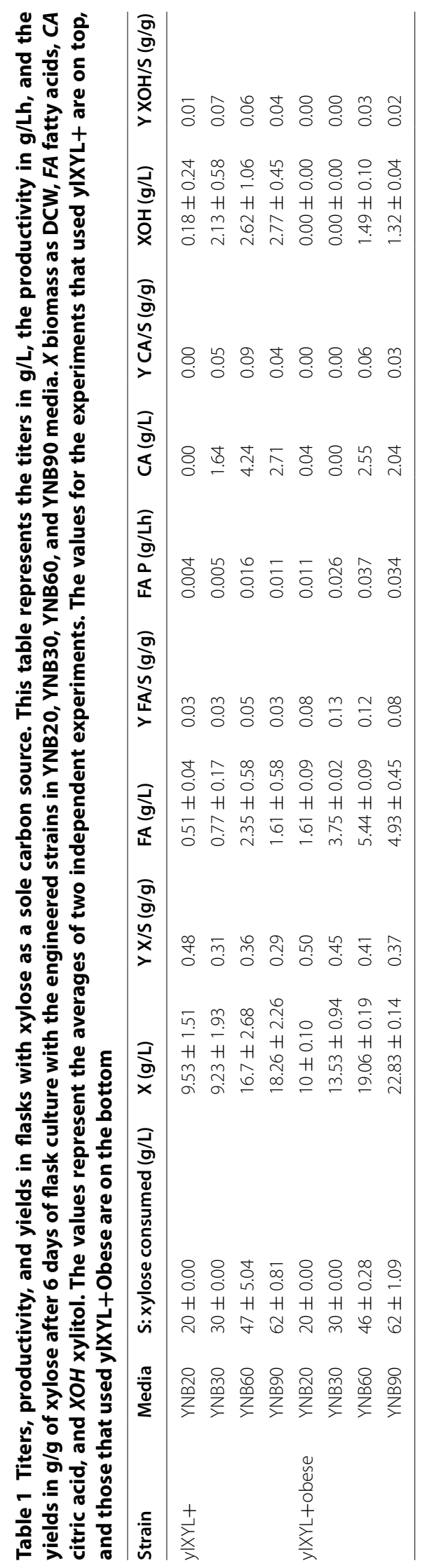


production of lipids, while ylXYL + can be beneficial for the synthesis of biotechnological products, namely xylitol and citric acid.

\section{$\mathrm{yIXYL}+$ and yIXYK+Obese are able to grow and produce lipids in lignocellulosic hydrolysate}

In this work, we obtained a lignocellulosic hydrolysate from agave bagasse, an abundant residue generated during the production of tequila $(1 \mathrm{~L}$ of tequila generates approximately $10 \mathrm{~kg}$ of bagasse). Analysis of the composition of this hydrolysate showed that its carbohydrate content $(87 \%$ of the DCW) is $15.5 \pm 7.4 \mathrm{~g} / \mathrm{L}$ glucose and $20.0 \pm 6.9 \mathrm{~g} / \mathrm{L}$ xylose and that it has acids (acetic acid $3.5 \pm 1.5 \mathrm{~g} / \mathrm{L}$, lactic acid $3.7 \pm 2.3$, and formic acid $0.6 \pm 0.3 \mathrm{~g} / \mathrm{L})$, glycerol $(1.5 \pm 0.7 \mathrm{~g} / \mathrm{L})$, and furfurals $(3.5 \pm 1.1 \mathrm{~g} / \mathrm{L})$.

We designed different culture media with different amounts of lignocellulosic hydrolysate $(10,20,30,40$, 50 , and $86 \%$ ) while keeping the concentrations of the phosphate buffer and the nitrogen source constant (see "Methods"). Therefore, an increasing amount of hydrolysate represents a higher $\mathrm{C} / \mathrm{N}$ ratio, but it also represents a higher amount of possible inhibitory compounds.

We found that ylXYL+ grew better with an increasing amount of hydrolysate, which seems to indicate a good tolerance to potentially toxic compounds (Fig. 3a). Generally, a higher $\mathrm{C} / \mathrm{N}$ ratio resulted in a higher lipid content (which oscillated from 4 to $7 \%$ ). Therefore, the total production of lipids was higher in the media with $86 \%$ hydrolysate.

ylXYL+Obese showed similar behavior to ylXYL+ (Fig. 3b); the produced biomass increased in parallel to the amount of hydrolysate as well as the lipid content. The lipid content increased from 11.5 to $24.5 \%$ of the DCW.
These results show that the modified $Y$. lipolytica strains are able to efficiently produce lipids directly from lignocellulosic hydrolysate.

\section{Metabolic engineering of xylose-consuming strains maximizes the conversion of sugars into lipids}

Acetyl-CoA is the precursor molecule for fatty acid synthesis. It can be converted to malonyl-CoA and, together, they can be used by the FAS complex (FAS1 and FAS2) to produce palmitic acid and stearic acid. In Y. lipolytica, the two major sources of acetyl-CoA are the enzyme $A C S 2$, which uses acetate as substrate, and ATP citrate lyase (heterodimer of $A C L 1$ and $A C L 2$ ), which uses citrate. However, other organisms have developed alternative pathways for acetyl-CoA production. Here, we have transferred two phosphoketolase pathways, named XP and XA here, which use xylulose-5-P, an intermediate in the xylose degradation pathway, as a substrate (Fig. 4a). The XP pathway is intended to convert xylulose-5-P to glyceraldehyde-3-P and acetyl-P via the XPKA enzyme (phosphoketolase) from Aspergillus nidulans and to convert the generated acetyl-P into acetyl-coA via PTA (phosphotransacetylase) from Bacillus subtilis. In the course of these experiments, the XP pathway increased fatty acid production from glucose in Y. lipolytica by $53 \%$ [23] and increased fatty acid production from xylose in the non-oleaginous fungus $A$. gossypii by 54\% [24]. The XA pathway expresses the XPKA enzyme to produce acetyl-P and the $A C K$ enzyme (acetate kinase) from $A$. nidulans to convert acetyl-P into acetate with the concomitant production of ATP. Thereafter, acetate can be converted into acetyl-CoA via the native $A C S 2$ enzyme.

We therefore generated strains ylXYL $+\mathrm{XP}$, ylXYL $+\mathrm{XA}$, ylXYL+Obese-XP, and ylXYL+Obese-XA and analyzed their capacity to produce lipids from xylose as a sole
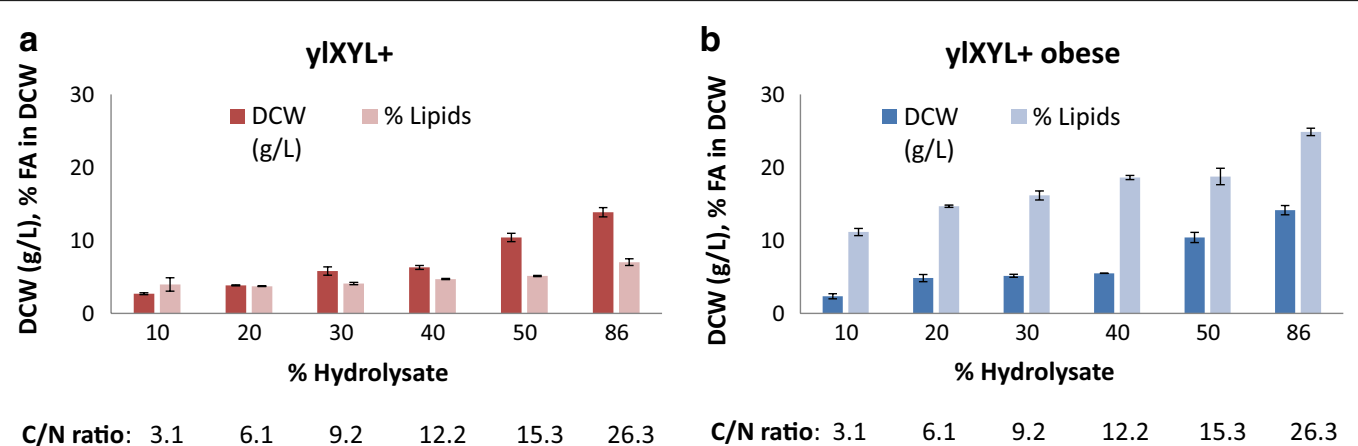

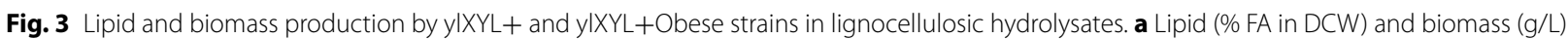
production in the $y I X Y L+$ strain when growing in media with different amounts of lignocellulosic hydrolysate (from 10 to $86 \%$ ). b Lipid (\% FA in $\mathrm{DCW}$ ) and biomass ( $\mathrm{g} / \mathrm{L}$ ) production in the $\mathrm{YIXYL+Obese} \mathrm{strain} \mathrm{when} \mathrm{growing} \mathrm{in} \mathrm{media} \mathrm{with} \mathrm{different} \mathrm{amounts} \mathrm{of} \mathrm{lignocellulosic} \mathrm{hydrolysate} \mathrm{(from}$ 10 to $86 \%$ ). The values represent the average values and the standard deviation of at least two replicates. The $\mathrm{C} / \mathrm{N}$ ratio for each condition is stated at the bottom of each bar 
a

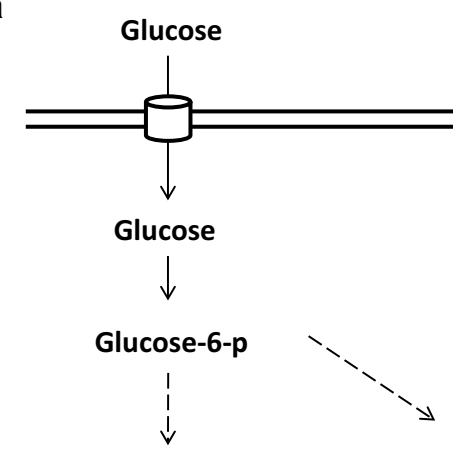

Glyceraldehyde-3-p

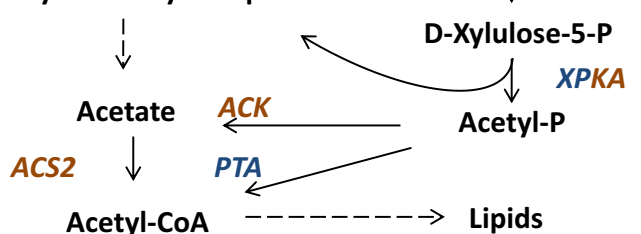

b

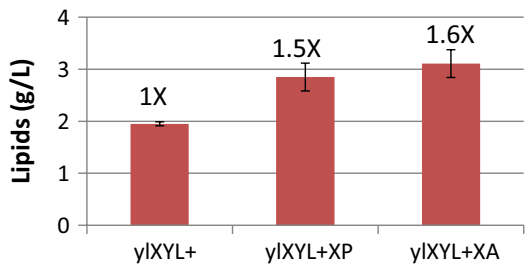

c

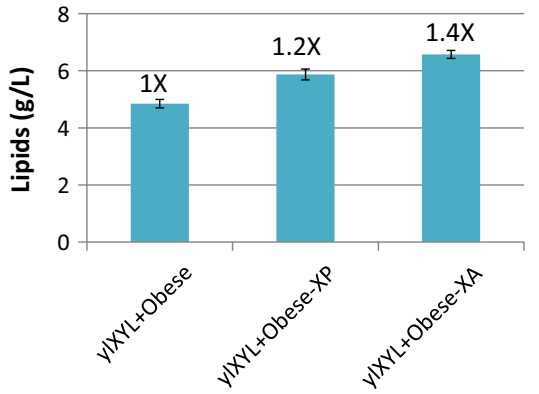

Fig. 4 Xylose utilization pathway enhanced for lipid production. a Schematic representation of the pathway leading to the consumption of xylose. $X R$ xylose reductase; $X D H$ xylitol dehydrogenase; and $X K$ xylulose kinase represent the xylose consumption pathway (in red). XPKA phosphoketolase, ACK acetate kinase, and ACS2 acetyl-CoA synthase represent the XA pathway (in brown), while XPKA phosphoketolase and PTA phosphotransacetylase are the XP pathway (in blue). Dashed lines represent multiple metabolic steps. The bar graphs represent the lipid production ( $\mathrm{g} / \mathrm{L}$ ) in the modified strains incorporating the XP or XA pathways compared to their parental strains, $y \mid X Y L+(\mathbf{b})$ and $y \mid X Y L+O b e s e ~(\mathbf{c})$, when they were grown on xylose as a sole carbon source. The values represent the average values and the standard deviation of at least two replicates. The fold increase in relation with the parental strain is indicated on the top of each bar

carbon source (media YNB60). The four strains showed a higher lipid production than their parental strains (Fig. 4b, c). Interestingly, the four strains were also able to produce a higher amount of biomass $(y l X Y L+X P$ $22 \%, y l X Y L+X A \quad 21 \%, y l X Y L+O b e s e-X P \quad 13 \%$, and ylXYL+Obese-XA 18\%) than their parental strains, and therefore, the lipid titers (grams of lipids per liter) were further increased. ylXYL $+X P$ and ylXYL $+X A$ produced 46 and 59\% more lipids than ylXYL+ (Fig. 4b), respectively, while ylXYL+Obese-XP and ylXYL+Obese-XA produced 21 and 35\% more lipids than ylXYL+Obese (Fig. 4c), respectively. We can here conclude that both strategies succeeded in the redirection of xylose towards acetyl-CoA and lipids, with slightly better results with the XA pathway. The higher efficiency of the XA pathway compared to the XP pathway had been reported for the production of fatty acid ethyl esters (FAEE) in S. cerevisiae from glucose [25]. We here selected ylXYL+Obese-XA as a good candidate to produce lipids from lignocellulosic hydrolysate in controlled conditions.

\section{Production of lipids in fed-batch fermentation from lignocellulosic hydrolysate in bioreactor-controlled conditions}

Based on the abovementioned experiments, ylXYL+Obese$\mathrm{XA}$, a strain overexpressing $X R, X D H$, and $X K$ for the utilization of xylose, XPKA and ACK for the conversion of xylulose-5-P into acetate, and DGA2 and GPD1 for boosting TAG production and deletion of POX1-6 and TGL4 in order to avoid the degradation of fatty acids and TAGs, showed the best behavior for the production of lipids from xylose, one of the most abundant sugars in the lignocellulosic hydrolysate from agave. We therefore decided to test its ability to produce lipids from lignocellulosic hydrolysate in 2-L bioreactors, together with a control strain (the wild type). We performed fed-batch fermentations with an initial C/N ratio of 15 (30 g of sugars, $18 \%$ glucose, and $12 \%$ xylose exclusively from the hydrolysate) to promote biomass formation followed by the controlled addition, after $24 \mathrm{~h}$, of 2 times concentrated hydrolysate at constant rate (60 $\mathrm{g}$ of sugars) in order to increase lipid production (overall $\mathrm{C} / \mathrm{N}$ of 45 ). The fermentations run for $96 \mathrm{~h}$, when all the sugars were consumed $(45 \mathrm{~g} / \mathrm{L})$ and the production of biomass and lipids were stable. Both glucose and xylose were consumed in the fermentations (wild type, Fig. 5a and ylXYL+Obese-XA, Fig. 5b), being glucose quickly depleted from the broth in $24 \mathrm{~h}$. As expected, xylose was degraded in both strains but much faster in ylXYL+ObeseXA (between 24 and $36 \mathrm{~h}$ ) than in the wild type (between 48 and $84 \mathrm{~h}$ ). Supplemented sugars in the feeding hydrolysate were consumed almost instantly, which promoted a longer steady state of biomass and lipid accumulation in 


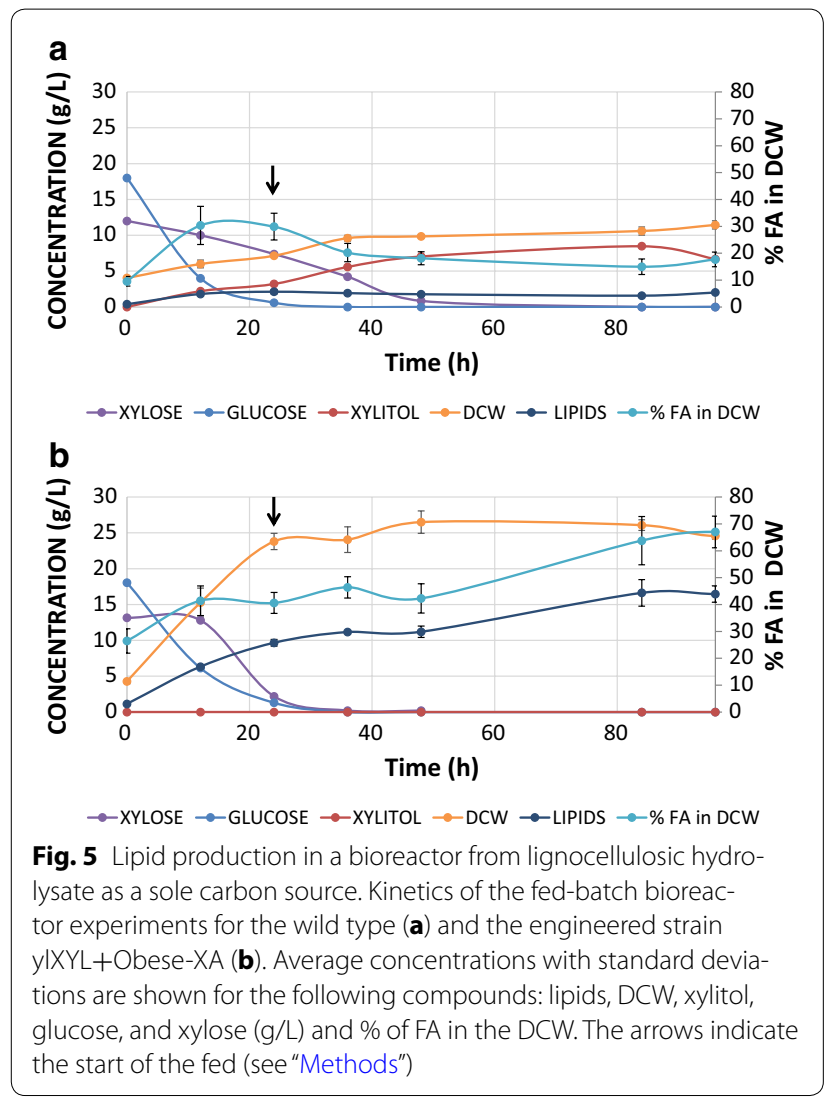

both strains. The biomass production was much higher in ylXYL+Obese-XA (25.8 g/L, compared to 11.5 in the wild type), presumably due to the efficient incorporation of the xylose. This is also supported by the fact that the wild type accumulates $8.5 \mathrm{~g} / \mathrm{L}$ of xylitol in the culture broth, originated by a non-complete degradation of xylose, while no xylitol is produced by the engineered strain. Similar behavior has been reported for the wild type growing in mixtures of glucose and xylose in flask [13]. Importantly, the lipid production reached $16.5 \mathrm{~g} / \mathrm{L}$ in the engineered strain, 8.3 times more than the wild type $(2 \mathrm{~g} / \mathrm{L})$. The maximum lipid content was $67 \%$ with a yield of $0.344 \mathrm{~g}$ lipids/g sugars and a maximum productivity of $0.185 \mathrm{~g} / \mathrm{L} / \mathrm{h}$. Interestingly, this is the highest yield ever reported for the production of lipids in Yarrowia lipolytica and it is equivalent to the maximum theoretical yield ( $0.364 \mathrm{~g}$ lipids/g glucose). Such high yield can be explained by the presence of other components in the hydrolysate that can be also consumed to generate more lipids and/or biomass. $5.3 \mathrm{~g} / \mathrm{L}$ of lactic acid, $2.4 \mathrm{~g} / \mathrm{L}$ of acetic acid, and $1 \mathrm{~g} / \mathrm{L}$ of glycerol present in the hydrolysate are degraded within 48,12 , and $12 \mathrm{~h}$, respectively (data not shown). Importantly, the high lipid yield and productivity is achieved from a very low-cost lignocellulosic hydrolysate. In future approaches, further bioreactor optimization in high cell densities could potentially improve the lipid titer.

\section{Conclusions}

In this work, we first showed that overexpression of the native $X D H, X R$, and $X K$ genes in Pold allowed it to grow in xylose as a sole carbon source. The same strategy was transferred to a strain that was modified to overproduce lipids and was able to efficiently convert xylose into oils. Importantly, this strain was still able to respond to the different $\mathrm{C} / \mathrm{N}$ ratios, and it showed proper growth in low-nitrogen media, which was not the case for Polf [20]. This feature allowed a boost in lipid production in culture media with a high $\mathrm{C} / \mathrm{N}$ ratio. We thereafter tested whether these strains were able to use agave lignocellulosic hydrolysate, which is mainly composed of xylose and glucose, as a carbon source for growth and oil production, regardless of the presence of potentially toxic agents. Moreover, we engineered two additional pathways to generate acetyl-CoA, a precursor of lipids, from xylulose-5-P, an intermediate in the degradation of xylose. We found that both pathways increased lipid production from xylose, and we selected the best strain to grow in fed-batch bioreactor-controlled conditions using cheap lignocellulosic hydrolysate as a unique carbon source. Importantly, the fermentation conditions achieved very high lipid yield and productivity, even higher than those described in expensive and defined synthetic media.

Therefore, this work serves as a proof of concept for the improvement of the production of lipids by $Y$. lipolytica from lignocellulosic materials. In addition, it also creates room for further improvements via bioreactor condition optimization, where alternative fed-batch or continuous processes can be explored. Moreover, the designed strains can be tested in other lignocellulosic hydrolysates with different carbohydrate compositions and varying amounts of potentially toxic compounds.

This work allows us to reduce the costs associated with the production of lipid-derived compounds, such as biofuels or chemicals, as well as the cost of producing other compounds, such as organic acids, proteins (highly produced by Y. lipolytica), or novel compounds derived from acetyl-CoA (which is enhanced in the described strategy). The results presented in this paper bring us one step closer to a feasible fermentation-based economy.

\section{Methods}

\section{Strains, cultures, and media}

The Y. lipolytica strains used in this study were Po1d $\left(\mathrm{Ura}^{-} \mathrm{Leu}^{-}\right)$[whose parental strain is the wild-type strain W29 (ATCC20460)] and its derived strains. The prototrophic strains generated in this study are listed in Additional file 1: Table S1. Media and growth conditions for Escherichia coli and $Y$. lipolytica have been described elsewhere [26]. Minimal medium contained 0.17\% (wt/ 
vol) yeast nitrogen base without amino acids and ammonium sulfate (YNBww, DIFCO); 2, 3, 6, or 9\% xylose (wt/vol; Merck, Fontenay-sous-Bois Cedex, France) for YNB20, YNB30, YNB60, and YNB90, respectively; $0.15 \%$ (wt $/ \mathrm{vol}$ ) $\mathrm{NH}_{4} \mathrm{Cl}$; and $50 \mathrm{mM}$ phosphate buffer ( $\mathrm{pH}$ 6.8). Media with glucose were prepared in a similar way as YNB by substituting $2 \%$ xylose with $2 \%$ glucose. Media containing both sugars included $3 \%$ glucose and $3 \%$ xylose. The media with hydrolysate contained 10,20 , $30,40,50$, and $86 \%(\mathrm{v} / \mathrm{v})$ of hydrolysate, $0.17 \%(\mathrm{wt} / \mathrm{vol})$ yeast nitrogen base (YNBww), 0.15\% (wt/vol) $\mathrm{NH}_{4} \mathrm{Cl}$, and $50 \mathrm{mM}$ phosphate buffer ( $\mathrm{pH}$ 6.8).

To evaluate lipid production from bagasse hydrolysates, batch bioreactor cultures were performed in duplicate in 3-L Applikon glass bioreactors using My Control consoles at $1 \mathrm{vvm}$ aeration, $750 \mathrm{rpm}$ agitation, and $\mathrm{pH} 6.8$. Working volume was $2 \mathrm{~L}$. Fed-batch cultures were performed following this strategy, beginning with $1 \mathrm{~L}$ of medium in a batch mode at the same conditions but using a concentration of $0.3 \%$ (wt/vol) $\mathrm{NH}_{4} \mathrm{Cl}$ in order to start at a $\mathrm{C} / \mathrm{N}$ ratio of 15 . After $24 \mathrm{~h} 1 \mathrm{~L}$ of fed medium containing $60 \mathrm{~g} / \mathrm{L}$ of total sugars from lignocellulosic hydrolysate, $0.17 \%(\mathrm{w} / \mathrm{v})$ YNBww and $50 \mathrm{mM}$ phosphate buffer ( $\mathrm{pH}$ 6.8) were added at a constant flow of $0.35 \mathrm{~mL} /$ $\mathrm{min}$. The hydrolysate was concentrated two times for the fed by evaporation. We did not concentration further the hydrolysate because it showed solid precipitations and thus associated changes in soluble composition.

\section{Lignocellulosic hydrolysate}

Agave bagasse was obtained from a tequila factory in Jalisco, Mexico. It was dried to a constant weight at $70{ }^{\circ} \mathrm{C}$ to measure its humidity prior to hydrolysis and characterization. Bagasse hydrolysis was performed through a chemical and enzymatic procedure according to patent application MX/E/2015/084126. Finally, a liquid hydrolysate was obtained. Furfurals, acids, alcohols, and sugar content in the liquid hydrolysate were measured by HPLC.

\section{Cloning and expression of heterologous and endogenous genes}

The endogenous genes $X D H$ (YALIOE12463), $X R$ (YALIOD07634), and XK (YALIF10923) were amplified from Pold genomic DNA using the appropriate primers (Additional file 1: Table S2). The PCR fragments were digested using BamHI/AvrII and were inserted into the plasmid JMP62 TEF [27] at the corresponding sites with an LEU2, URA3, or HYG marker. The heterologous genes XPKA (AN4913.2) and ACK (AN4914.2) from $A$. nidulans and PTA (AIY95081.1) from B. subtilis were codon optimized, synthesized by GenScript, and cloned into JMP62 TEF-derived plasmids with a URA or LEU marker. The complete list of genes used in this study is shown in Additional file 1: Table S3.

Expression vectors were digested with NotI, purified on a gel, and used for transformation as described in previous works [28]. Depending on their genotype, transformants were selected on YNBG ( $1 \%$ glucose) media, supplemented with uracil or leucine (for auxotrophic strains), or on YPDHyg. Positive transformants were checked by PCR. The removal of the selection marker was carried out via the LoxP-Cre system, which is widely used in Yarrowia [29]. The Pold (JMY195) strain transformed with $y l X R, y l X D H$, and $y l X K$ was named ylXYL+, while the obese strain JMY3501 [14], which is highly modified for enhancing lipid accumulation, was further transformed with the same xylose pathway genes and was named ylXYL+Obese.

Restriction enzymes were obtained from OZYME (Saint-Quentin-en-Yvelines, France). PCR amplifications were performed in an Eppendorf 2720 thermal cycler with GoTaq DNA polymerases (Promega). PCR fragments were purified with a Qiagen Purification Kit (Qiagen, Hilden, Germany). All reactions were performed according to the manufacturers' instructions.

\section{Determination of DCW, growth rate, sugars, acids, and alcohols}

To determine DCW in the flask experiments, $2 \mathrm{~mL}$ of culture was washed and lyophilized in a pre-weighed tube. The differences in weight corresponded to the $\mathrm{mg}$ of cells found in $2 \mathrm{~mL}$ of culture.

Growth tests were performed in $100 \mu \mathrm{L}$ cultures in 96-well plates, with constant shaking, in the presence of $0.5 \%$ glucose or xylose as a carbon source. Growth was monitored by measuring the optical density $\left(\mathrm{OD}_{600 \mathrm{~nm}}\right)$ at different intervals with a microtiter plate reader (Biotek, Colmar, France). For each strain and set of conditions, we used at least two biological replicates. A $2 \mathrm{~mL}$ preculture was grown for $16 \mathrm{~h}$ and was used to inoculate the cultures at an OD600 of 0.2. The growth rate was calculated in the exponential phase for each strain and condition. The lag phase was determined as the time at which the exponential phase began.

Glucose, xylose, xylitol, and citric acid were identified and quantified by HPLC (UltiMate 3000, Dionex-Thermo Fisher Scientific, UK) using an Aminex HPX87H column coupled to UV $(210 \mathrm{~nm})$ and RI detectors. The column was eluted with $0.01 \mathrm{~N} \mathrm{H}_{2} \mathrm{SO}_{4}$ at room temperature and a flow rate of $0.6 \mathrm{~mL} / \mathrm{min}$. Identification and quantification were achieved via comparisons to standards. Before being subjected to HPLC analysis, samples were filtered on membranes of $0.45-\mu \mathrm{m}$ pore size. Citric acid and isocitric acid are undistinguishable in our methods and thus when citric acid is referred in the text the value represents the sum of all citric acid forms. 


\section{Lipid quantification}

Lipids from aliquots of $10-20 \mathrm{mg}$ of cells were converted into their methyl esters with freeze-dried cells according to Browse et al. [30] and were used for gas chromatography (GC) analysis. GC analysis of fatty acid (FA) methyl esters was performed with a Varian 3900 instrument equipped with a flame ionization detector and a Varian FactorFour vf- $23 \mathrm{~ms}$ column, where the bleed specification at $260{ }^{\circ} \mathrm{C}$ is $3 \mathrm{pA}(30 \mathrm{~m}, 0.25 \mathrm{~mm}, 0.25 \mu \mathrm{m})$. FAs were identified by comparison to commercial FA methyl ester standards (FAME32; Supelco) and quantified by the internal standard method, which involves the addition of $50 \mu \mathrm{g}$ of commercial C17:0 (Sigma).

\section{Microscopic analysis}

The image was acquired using a Zeiss Axio Imager M2 microscope (Zeiss, Le Pecq, France) with a $100 \times$ objective and Zeiss filters 45 and 46 for fluorescence microscopy. Axiovision 4.8 software (Zeiss, Le Pecq, France) was used for image acquisition. Lipid body visualization was performed after adding Bodipy ${ }^{\circledR}$ Lipid Probe $(2.5 \mathrm{mg} / \mathrm{mL}$ in ethanol; Invitrogen) to the cell suspension $\left(A_{600}\right.$ of 5$)$ with incubation for $10 \mathrm{~min}$ at room temperature.

\section{Additional file}

Additional file 1: Figure S1. Growth curves of Po1d (in glucose and xylose media), ylXYL+ and ssXYL+ (in xylose media) when grown on microplate ( $0.5 \%$ of carbon source). Figure S2. Production of xylitol and citric acid by the modified strains grown on xylose as a sole carbon source. Values of the production of xylitol (red) and citric acid (green) when the strains $y \mid X Y L+(A)$ or $y \mid X Y L+O b e s e(B)$ were grown in media with different concentrations of xylose (YNB20, YNB30, YNB60, and YNB90; see "Methods"). The values represent the average values and the standard deviation of at least two replicates. Table S1. List of prototrophic strains used in this study. Table S2. List of primers used in this study. Table $\mathbf{S 3 .}$ List of genes modified in this study.

\section{Abbreviations \\ TAG: triacylglycerol; FAEE: fatty acid methyl ester; DCW: dry cell weight; XR: xylose reductase; $\mathrm{XDH}$ : $x y$ litol dehydrogenase; XK: xylulose kinase; XPKA: phosphoketolase; ACK: acetate kinase; ACS2: acetyl-CoA synthase; C/N: ration carbon to nitrogen; $C 5$ : pentose.}

\section{Authors' contributions}

RLA and XN designed and performed most of the experiments. RLA conceived the study. RLA and JMN directed and supervised the work. RLA wrote the first draft of the manuscript. RLA, GS, AML, and JMN revised the manuscript. All authors read and approved the final manuscript.

\section{Author details}

${ }^{1}$ Micalis Institute, INRA, AgroParisTech, Université Paris-Saclay, 78350 Jouy-en-Josas, France. ${ }^{2}$ Industrial Biotechnology, Centro de Investigación y Asistencia en Tecnología y Diseño del Estado de Jalisco (CIATEJ) A.C., 44270 Guadalajara, Jalisco, Mexico. ${ }^{3}$ Department of Bioengineering, Imperial College London, London SW7 2AZ, UK.

\section{Acknowledgements and Funding}

This work has benefited from aid from the Agence Nationale de la Recherche under the "Investissements d'avenir" program with reference code ANR-11BTBR-0003 and from project FSE-250014 and FSE-248090 (CONACYT-SENER,
Mexico). R. Ledesma-Amaro received financial support from the European Union through the Marie-Curie FP7 COFUND People Program in the form of an AgreenSkills Fellowship and from Imperial College London in the form of a IC Research Fellowship. X. Niehus is the recipient of a Ph.D. scholarship from the Consejo Nacional de Ciencia y Tecnología (336376/234603) in Mexico (CONACYT).

\section{Availability of data and materials}

All data generated or analyzed during this study are included in this published article and its supplementary information files.

\section{Consent for publication}

Not applicable.

Ethics approval and consent to participate

Not applicable.

\section{Competing interests}

The authors declare that they have no competing interests.

\section{Publisher's Note}

Springer Nature remains neutral with regard to jurisdictional claims in published maps and institutional affiliations.

Received: 23 June 2017 Accepted: 8 January 2018

Published online: 22 January 2018

\section{References}

1. Ledesma-Amaro R, Nicaud JM. Yarrowia lipolytica as a biotechnological chassis to produce usual and unusual fatty acids. Prog Lipid Res. 2015;61:40-50. https://doi.org/10.1016/j.plipres.2015.12.001.

2. Dulermo T, Nicaud JM. Involvement of the G3P shuttle and beta-oxidation pathway in the control of TAG synthesis and lipid accumulation in Yarrowia lipolytica. Metab Eng. 2011;13:482-91. https://doi.org/10.1016/j. ymben.2011.05.002.

3. Tai M, Stephanopoulos G. Engineering the push and pull of lipid biosynthesis in oleaginous yeast Yarrowia lipolytica for biofuel production. Metab Eng. 2013;15:1-9. https://doi.org/10.1016/j.ymben.2012.08.007.

4. Qiao K, Wasylenko TM, Zhou K, Xu P, Stephanopoulos G. Lipid production in Yarrowia lipolytica is maximized by engineering cytosolic redox metabolism. Nat Biotechnol. 2017;35:173-7. https://doi.org/10.1038/nbt.3763.

5. Dulermo T, Treton B, Beopoulos A, Kabran Gnankon AP, Haddouche R, Nicaud JM. Characterization of the two intracellular lipases of $Y$. lipolytica encoded by TGL3 and TGL4 genes: new insights into the role of intracellular lipases and lipid body organisation. Biochim Biophys Acta. 2013;1831:1486-95. https://doi.org/10.1016/j.bbalip.2013.07.001.

6. Blazeck J, Hill A, Liu L, Knight R, Miller J, Pan A, et al. Harnessing Yarrowia lipolytica lipogenesis to create a platform for lipid and biofuel production. Nat Commun. 2014;5:3131. https://doi.org/10.1038/ncomms4131.

7. Qiao K, Imam Abidi SH, Liu H, Zhang H, Chakraborty S, Watson N, et al. Engineering lipid overproduction in the oleaginous yeast Yarrowia lipo/ytica. Metab Eng. 2015. https://doi.org/10.1016/j.ymben.2015.02.005.

8. Ledesma-Amaro R, Dulermo R, Niehus X, Nicaud JM. Combining metabolic engineering and process optimization to improve production and secretion of fatty acids. Metab Eng. 2016;38:38-46. https://doi. org/10.1016/j.ymben.2016.06.004.

9. Shaw AJ, Lam FH, Hamilton M, Consiglio A, MacEwen K, Brevnova EE, et al. Metabolic engineering of microbial competitive advantage for industrial fermentation processes. Science. 2016;353:583-6. https://doi. org/10.1126/science.aaf6159.

10. Beopoulos A, Verbeke J, Bordes F, Guicherd M, Bressy M, Marty A, et al. Metabolic engineering for ricinoleic acid production in the oleaginous yeast Yarrowia lipolytica. Appl Microbiol Biotechnol. 2014;98:251-62. https://doi.org/10.1007/s00253-013-5295-X.

11. Wang G, Xiong X, Ghogare R, Wang P, Meng Y, Chen S. Exploring fatty alcohol-producing capability of Yarrowia lipolytica. Biotechnol Biofuels. 2016;9:107. https://doi.org/10.1186/s13068-016-0512-3. 
12. Ledesma-Amaro R, Dulermo T, Nicaud JM. Engineering Yarrowia lipolytica to produce biodiesel from raw starch. Biotechnol Biofuels. 2015;8:148. https://doi.org/10.1186/s13068-015-0335-7.

13. Ledesma-Amaro R, Lazar Z, Rakicka M, Guo Z, Fouchard F, Coq AC, et al. Metabolic engineering of Yarrowia lipolytica to produce chemicals and fuels from xylose. Metab Eng. 2016. https://doi.org/10.1016/j. ymben.2016.07.001.

14. Lazar Z, Dulermo T, Neuveglise C, Crutz-Le Coq AM, Nicaud JM. Hexokinase-A limiting factor in lipid production from fructose in Yarrowia lipolytica. Metab Eng. 2014;26C:89-99. https://doi.org/10.1016/j. ymben.2014.09.008.

15. Lazar Z, Gamboa-Melendez H, Le Coq AC, Neuveglise C, Nicaud JM. Awakening the endogenous Leloir pathway for efficient galactose utilization by Yarrowia lipolytica. Biotechnol Biofuels. 2015;8:185. https://doi. org/10.1186/s13068-015-0370-4.

16. Ledesma-Amaro R, Nicaud JM. Metabolic engineering for expanding the substrate range of Yarrowia lipolytica. Trends Biotechnol. 2016. https://doi org/10.1016/j.tibtech.2016.04.010.

17. Jonsson $\sqcup$, Alriksson B, Nilvebrant NO. Bioconversion of lignocellulose: inhibitors and detoxification. Biotechnol Biofuels. 2013;6:16. https://doi. org/10.1186/1754-6834-6-16.

18. Anwar Z, Gulfraz M, Irshad M. Agro-industrial lignocellulosic biomass a key to unlock the future bio-energy: a brief review. J Radiat Res Appl Sci. 2014;7:163-73. https://doi.org/10.1016/j.jrras.2014.02.003.

19. Li H, Alper HS. Enabling xylose utilization in Yarrowia lipolytica for lipid production. Biotechnol J. 2016;11:1230-40. https://doi.org/10.1002/ biot.201600210.

20. Rodriguez GM, Hussain MS, Gambill L, Gao D, Yaguchi A, Blenner M. Engineering xylose utilization in Yarrowia lipolytica by understanding its cryptic xylose pathway. Biotechnol Biofuels. 2016;9:149. https://doi org/10.1186/s13068-016-0562-6.

21. Tsigie YA, Wang CY, Kasim NS, Diem QD, Huynh LH, Ho QP, et al. Oil production from Yarrowia lipolytica Po1g using rice bran hydrolysate. J Biomed Biotechnol. 2012;2012:378384. https://doi. org/10.1155/2012/378384.
22. Tsigie YA, Wang CY, Truong CT, Ju YH. Lipid production from Yarrowia lipolytica Po1g grown in sugarcane bagasse hydrolysate. Bioresour Technol. 2011;102:9216-22. https://doi.org/10.1016/j.biortech.2011.06.047.

23. Xu P, Qiao K, Ahn WS, Stephanopoulos G. Engineering Yarrowia lipolytica as a platform for synthesis of drop-in transportation fuels and oleochemicals. Proc Natl Acad Sci USA. 2016;113:10848-53. https://doi.org/10.1073/ pnas.1607295113.

24. Diaz-Fernandez D, Lozano-Martinez P, Buey RM, Revuelta JL, Jimenez A. Utilization of xylose by engineered strains of Ashbya gossypii for the production of microbial oils. Biotechnol Biofuels. 2017;10:3. https://doi. org/10.1186/s13068-016-0685-9.

25. de Jong BW, Shi S, Siewers V, Nielsen J. Improved production of fatty acid ethyl esters in Saccharomyces cerevisiae through up-regulation of the ethanol degradation pathway and expression of the heterologous phosphoketolase pathway. Microb Cell Fact. 2014;13:39. https://doi. org/10.1186/1475-2859-13-39.

26. Dulermo R, Gamboa-Melendez H, Ledesma-Amaro R, Thevenieau F, Nicaud JM. Unraveling fatty acid transport and activation mechanisms in Yarrowia lipolytica. Biochim Biophys Acta. 2015. https://doi.org/10.1016/j. bbalip.2015.04.004.

27. Dulermo R, Brunel F, Dulermo T, Ledesma-Amaro R, Vion J, Trassaert $M$, et al. Using a vector pool containing variable-strength promoters to optimize protein production in Yarrowia lipolytica. Microb Cell Fact. 2017;16:31. https://doi.org/10.1186/s12934-017-0647-3.

28. Gajdos P, Ledesma-Amaro R, Nicaud JM, Certik M, Rossignol T. Overexpression of diacylglycerol acyltransferase in Yarrowia lipolytica affects lipid body size, number and distribution. FEMS Yeast Res. 2016. https://doi. org/10.1093/femsyr/fow062.

29. Fickers P, Le Dall MT, Gaillardin C, Thonart P, Nicaud JM. New disruption cassettes for rapid gene disruption and marker rescue in the yeast Yarrowia lipolytica. J Microbiol Methods. 2003;55:727-37.

30. Browse J, McCourt PJ, Somerville CR. Fatty acid composition of leaf lipids determined after combined digestion and fatty acid methyl ester formation from fresh tissue. Anal Biochem. 1986;152:141-5.

\section{Submit your next manuscript to BioMed Central and we will help you at every step:}

- We accept pre-submission inquiries

- Our selector tool helps you to find the most relevant journal

- We provide round the clock customer support

- Convenient online submission

- Thorough peer review

- Inclusion in PubMed and all major indexing services

- Maximum visibility for your research

Submit your manuscript at www.biomedcentral.com/submit
() Biomed Central 